\title{
PSEUDO CLOSED TRAJECTORIES IN THE FAMILY OF TRAJECTORIES DEFINED BY A SYSTEM OF DIFFERENTIAL EQUATIONS*
}

\author{
BY \\ L. A. MACCOLL \\ Bell Telephone Laboratories
}

1. Introduction. This paper is concerned with certain simple closed curves, here called pseudo closed trajectories, which play an important part in determining the topological properties of the family of trajectories (or characteristics) defined by a system of differential equations of the form

$$
\frac{d x}{d t}=X(x, y), \quad \frac{d y}{d t}=Y(x, y) .
$$

Some of these curves are considered in a rather incidental way in the writings of Poincaré.** However, the full concept of pseudo closed trajectories does not seem to have been discussed explicitly heretofore.

We assume that all of the variables in the equations (1) are real, that the functions $X$ and $Y$ are continuous in an open connected region $R$ in the $x y$-plane, that these functions satisfy Lipschitz conditions locally throughout $R$, and that the real curves $X=0$ and $Y=0$, if they exist, have only simple intersections. We assume that these points of intersection have no point of condensation in the region $R$. We also assume that if the functions $X$ and $Y$ are not linear, they satisfy conditions which are sufficient to insure that the topological properties of the family of trajectories in a small neighborhood of any singular point (point of intersection of the curves $X=0, Y=0$ ) other than a center are the same as in the case obtained by replacing $X$ and $Y$ by their linear approximations. The last assumption is satisfied if $X$ and $Y$ are, for instance, of class $C^{2}$ in $R$.

In brief, our assumptions are simply the ones that are usually employed in discussions of the family of trajectories. The necessary information about the implications of the assumptions is readily available in the literature. $\dagger$

Since the notion of a trajectory is a familiar one, we have used the term so far without any explanations. However, in order to avoid the danger of future ambiguities, it will be well now to define the term explicitly.

Let $x=\varphi(t), y=\psi(t)$ be a solution of the system of equations (1). It is understood that the solution is defined over a certain maximum (finite or infinite) open interval $I^{(t)}$ on the $t$-axis.

As $t$ describes $I^{(t)}$ once in the sense of increasing $t$, the point $(x, y)=(\varphi(t), \psi(t))$ describes a certain curve $T$ in $R$. We call $T$ a trajectory.

*Received Aug. 3, 1949.

**See, for instance, Oeuvres de Henri Poincaré, t. I, p. 56 et seq. Poincaré's considerations concerning these curves are limited to pseudo closed trajectories which have only exterior vertices or only interior vertices, and to the possibility of trajectories being asymptotic to such pseudo closed trajectories. Exterior and interior vertices are defined in the next section.

†A good discussion, covering most of the points of interest, is given in the recent book by Tricomi, Equazioni differenziali, Einaudi, Torino, 1948. 
Any singular point is itself a trajectory, a point trajectory; and these are the only point trajectories. A trajectory $T$ which is not a point trajectory is oriented, the positive sense on $T$ being that in which the curve is described as $t$ increases.

2. Pseudo closed trajectories. Under our assumptions we may have just four kinds of singular points: nodes, foci, centers and saddle points. Since the topological properties of the family of trajectories in the neighborhood of any one of these singular points are well known, it is unnecessary to review them extensively here.

We recall that a node or focus is said to be stable or unstable according as the oriented arcs of trajectories in the neighborhood proceed toward or away from the singular point.

In the neighborhood of a saddle point there are just two arcs of trajectories which proceed toward the saddle point. These arcs, considered for the moment as being unoriented, together with the saddle point itself, form a smooth arc. Also, there are just two arcs of trajectories which proceed away from the saddle point; and these, together with the saddle point, form a smooth arc. The two smooth arcs described have distinct tangents at the saddle point. These phenomena are illustrated in the typical Fig. 1.

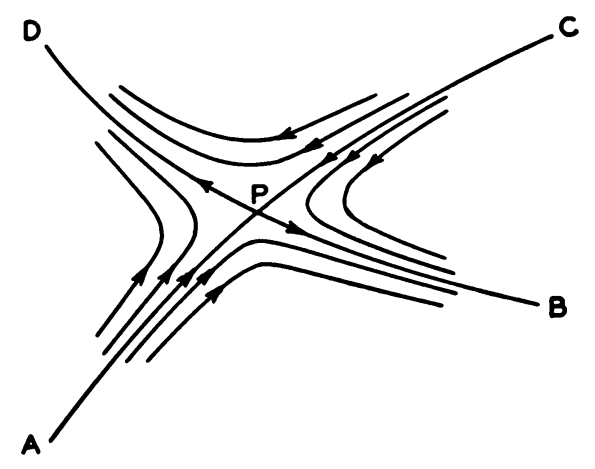

FIG. 1.

Elementary examples show that a trajectory may proceed from a saddle point to the same or a different saddle point.* We now assume that we have a case in which certain trajectories of this kind exist. Let the symbols $T_{1}, T_{2}, \cdots$ denote these trajectories.

Using a finite set of the trajectories $T_{i}$, and also using saddle points as necessary to connect these trajectories, we try to construct a closed curve $\Gamma$ satisfying the following conditions:

$C_{1}: \quad \Gamma$ is oriented, the orientation being that given by the orientations of the trajectories $T_{i}$ contained in $\Gamma$.

$C_{2}: \quad \Gamma$ is a simple closed curve.

Thus, referring to Fig. 1, $C_{1}$ implies that if $A P$ is an arc of $\Gamma$, the succeeding arc is $P B$ or $P D$. Also, if $A P$ is an arc of $\Gamma, C_{2}$ implies that the arc $C P$ does not belong to $\Gamma$.

We shall call a curve $\Gamma$ satisfying the above conditions a pseudo closed trajectory, or briefly a p.c.t. There are families of trajectories which contain no p.c.t. On the other hand, familiar or easily constructed examples show that there are cases in which p.c.t. do exist. In all of the following we assume that we have a case of the latter kind.

*See, for instance, Andronow and Chaikin, Theory of oscillations, Princeton, 1949, p. 71. 
We observe that different p.c.t. may have isolated points, or entire arcs, in common.

We shall call the saddle points which lie on a p.c.t. the vertices of the p.c.t. It is necessary to draw a distinction between two kind of vertices, which we call exterior vertices and interior vertices, respectively.

Let $\Gamma$ be a p.c.t., and $P$ one of its vertices. As a Jordan curve, $\Gamma$ divides its point set complement in the plane into a exterior region and an interior region. In the neighborhood of $P$ we have an arc of trajectory proceeding toward $P$, and an arc of trajectory proceeding from $P$, neither of these arcs belonging to $\Gamma$. If both of these arcs of trajectories are in the exterior region, we say that $P$ is an exterior vertex; and if both of these arcs are in the interior region, we say that $P$ is an interior vertex.* In the case of the p.c.t. $\Gamma$ shown in Fig. 2, $P_{1}$ and $P_{2}$ are interior vertices, and $P_{3}$ is an exterior vertex.

\section{The fundamental theorem concerning pseudo closed trajectories.}

TheOREM. Let $\Gamma$ be a p.c.t. such that all points of the interior region determined by $\Gamma$ belong to $R$. Let $N_{n}, N_{f}, N_{c}, N_{s}$, respectively, denote the numbers of nodes, foci, centers, and saddle points in the interior region, and let $N_{i v}$ denote the number of interior vertices of $\Gamma$. Then we have the relation

$$
N_{n}+N_{f}+N_{c}-N_{s}=1+N_{\text {iv }} .
$$

The proof of this theorem depends upon the following two familiar theorems.

$T_{1}$ : Let $\Gamma$ be a simple closed plane curve consisting of a finite set of smooth arcs meeting at corners. As the running point describes $\Gamma$ once in the counterclockwise sense, the tangent rotates through $+2 \pi$ radians, it being understood that at an exterior (interior) corner the tangent rotates counterclockwise (clockwise) through the appropriate exterior (interior) angle. $\dagger$

$T_{2}$ : The Poincaré index** of a simple closed curve which does not pass through any singular point, and which is such that the interior region determined by the curve belongs to $R$, is equal to the sum of the numbers of nodes, foci, and centers in the interior region, diminished by the number of saddle points in the interior region.

Let $\Gamma$ be a p.c.t. with interior vertices $P_{1}, \cdots, P_{k}$. It is understood that these points are ranged in the order in which they are encountered when $\Gamma$ is described in the sense of its natural orientation.

We choose points (not vertices) $Q_{1}^{\prime}, Q_{1}^{\prime \prime}, \cdots, Q_{k}^{\prime}, Q_{k}^{\prime \prime}$ on $\Gamma$ so that the typical arc $Q_{i}^{\prime} Q_{i}^{\prime \prime}$ of $\Gamma$ contains $P_{i}$, and no other vertex. At each of the points $Q_{i}^{\prime}, Q_{i}^{\prime \prime}$ we draw the normal to $\Gamma$.

Now we construct a simple closed curve $\Gamma^{*}$ as follows.

(1) For each $i$, we join a point on the exterior normal at $Q_{i}^{\prime}$ to a point on the exterior normal at $Q_{i}^{\prime \prime}$ by means of an arc of trajectory. The classical continuity theorems, together with the known behavior of the trajectories in the neighborhood of a saddle point, insure that this construction is possible, provided that the initial point of the arc of trajectory is taken sufficiently near to $\Gamma$.

\footnotetext{
*It is clear that it is impossible for one of these arcs to be in the exterior region and the other in the interior region.

$\dagger \mathrm{A}$ rigorous modern proof of this intuitively evident theorem is given by J. Lifshitz, Boletin de la Sociedad Matematica Mexicana, 3, 21-25 (1946).

${ }^{* *}$ Following the usual modern practice, we reckon the indices as the negatives of the indices actually defined by Poincaré.
} 
(2) For $i=1, \cdots, k-1$, we join a point on the interior normal at $Q_{i}^{\prime \prime}$ to a point on the interior normal at $Q_{i+1}^{\prime}$ by means of an arc of trajectory. If the initial point of the arc is taken sufficiently near $\Gamma$, this construction is possible, for the reasons mentioned above. Similarly, we join a point on the interior normal at $Q_{k}^{\prime \prime}$ to a point on the interior normal at $Q_{1}^{\prime}$ by an arc of trajectory.

(3) We complete $\Gamma^{*}$ by joining the end points of the arcs of trajectories that we have drawn by short segments of the normals to $\Gamma$ at the points $Q_{i}^{\prime}, Q_{i}^{\prime \prime}$.

For the sake of greater clarity, we emphasize the fact that the curve $\Gamma^{*}$ is supposed to be constructed so that each arc of trajectory belonging to $\Gamma^{*}$ lies very close to the corresponding arc of $\Gamma$ (i.e. an $\operatorname{arc} Q_{i}^{\prime} Q_{i}^{\prime \prime}$, an $\operatorname{arc} Q_{i}^{\prime \prime} Q_{i+1}^{\prime}$, or the $\operatorname{arc} Q_{k}^{\prime \prime} Q_{i}^{\prime}$ ) throughout its length. This is possible, because we have constructed the curve so as to avoid the arcs of trajectories which proceed to and from the vertices of $\Gamma$, and which do not belong to $\Gamma$.

Fig. 2 illustrates the construction of $\Gamma^{*}$ in a simple case.

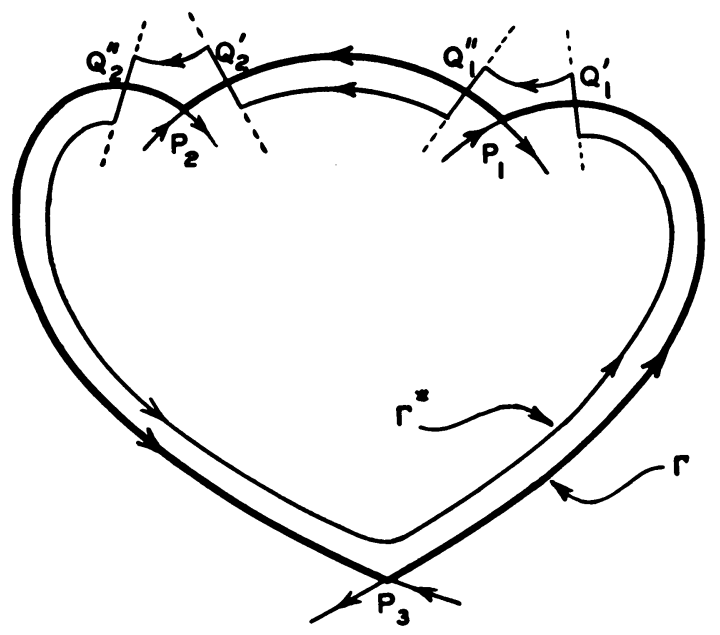

FIG. 2.

The curve $\Gamma^{*}$ is closed, by its construction. A little consideration suffices to show that if the segments of normals to $\Gamma$ belonging to $\Gamma^{*}$ are taken sufficiently short, $\Gamma^{*}$ is a simple closed curve, and that the interior region determined by $\Gamma^{*}$ belongs to $R$. Also, under the same condition, $\Gamma^{*}$ does not pass through any singular point; and the singular points contained in the interior region determined by $\Gamma^{*}$ are the singular points contained in the interior region determined by $\Gamma$, plus the interior vertices of $\Gamma$. Therefore, by $T_{2}$ above, the index of $\Gamma^{*}$ is $N_{n}+N_{f}+N_{c}-N_{s}-N_{i v}$.

We now proceed to evaluate the index of $\Gamma^{*}$ in a different way.

The index of $\Gamma^{*}$ is the net number of revolutions performed by the field vector $(X, Y)$ in the positive sense when the point $(x, y)$ describes $\Gamma^{*}$ once counterclockwise. The continuity of the functions $X, Y$ implies that the contribution to the index, conceived in this way, coming from the short segments of normals to $\Gamma$ is small in absolute value. Now consider the contribution coming from the arcs of trajectories belonging to $\Gamma^{*}$.

We note that at a point of $\Gamma$, other than a vertex, the field vector does not vanish, 
and has the direction of the tangent to $\Gamma$. From this, and the continuity of $X$ and $Y$, it follows at once that the contribution to the index of $\Gamma^{*}$ coming from the arcs of trajectories belonging to $\Gamma^{*}$ is nearly equal to the net number of revolutions performed by the tangent to $\Gamma$ when the point of tangency describes $\Gamma$ once counterclockwise. By $T_{1}$ above, this number of revolutions is +1 .

The conclusions of the preceding two paragraphs imply that the index of $\Gamma^{*}$ cannot be far different from +1 . Hence, since the index is necessarily an integer, the index is +1 exactly.

When we equate the two expressions for the index that we have obtained, we have the conclusion of the theorem.

The preceding reasoning is based upon the hypothesis that $\Gamma$ has at least two interior vertices. However, the same proof, with some slight and obvious modifications of the expository details, applies also when $\Gamma$ has only one interior vertex.

If $\Gamma$ has no interior vertices, we draw the normal to $\Gamma$ at a point $Q$ which is not a vertex. Then we construct a simple closed curve $\Gamma^{*}$ out of a short segment of the interior normal and an arc of trajectory which joins the end points of the segment, and which lies very close to $\Gamma$ throughout its length. Having constructed $\Gamma^{*}$ in this way, we obtain the conclusion of the theorem by means of reasoning which is essentially similar to that used above.

4. Study of some simple cases. In the case of a p.c.t. that has no interior vertices, we have the relation

$$
N_{n}+N_{f}+N_{c}-N_{\mathrm{s}}=1 .
$$

This is the same as the relation that we have in the case of a closed trajectory (in the ordinary strict sense). Consequently, the existence of a p.c.t. without interior vertices leads to essentially the same conclusions, in regards to the topological properties of the family of trajectories in the interior region, that the existence of a closed trajectory does. Since these conclusions have been thoroughly discussed in the literature, we shall not consider them here. Instead, we proceed to consider some simple cases in which we have p.c.t. with interior vertices.

\section{CASE 1.}

Suppose that $\Gamma$ is a p.c.t. with just one interior vertex $P$, and suppose that there are no centers, saddle points, or closed trajectories in the interior region determined by $\Gamma$. (It is understood that the interior region belongs to $R$.)

The fundamental theorem implies that there are just two singular points in the interior region. Either of these points, which we shall denote by the symbols $S_{1}, S_{2}$, may be a node or a focus. We shall see that when the types of these singular points are determined, there remain just two topological possibilities as to the structure of the family of trajectories in the interior region.

In the interior region there is one arc of trajectory which proceeds from $P$, and one arc of trajectory which proceeds to $P$. In the first subcase, illustrated by Fig. 3a, these arcs belong to the same trajectory $T$. The trajectory $T$ and the saddle point $P$ form a p.c.t. $\Gamma^{\prime}$ with just one vertex, which is exterior. The interior region determined by $\Gamma^{\prime}$ is contained in the interior region determined by $\Gamma$. The fundamental theorem implies that one of the points $S_{1}, S_{2}$, say the point $S_{1}$, lies in the interior region determined 
by $\Gamma^{\prime}$. Then $S_{2}$ must lie in the complement of this region with respect to the interior region determined by $\Gamma$.

Suppose that the arcs of trajectories which proceed from and to $P$, respectively, do not belong to the same trajectory. Then the arc of trajectory proceeding from $P$ cannot proceed to any singular point on $\Gamma$, for all of the arcs of trajectories proceeding to such singular points are otherwise accounted for. The arc of trajectory cannot approach asymptotically to a closed trajectory, for there are no such trajectories in the interior

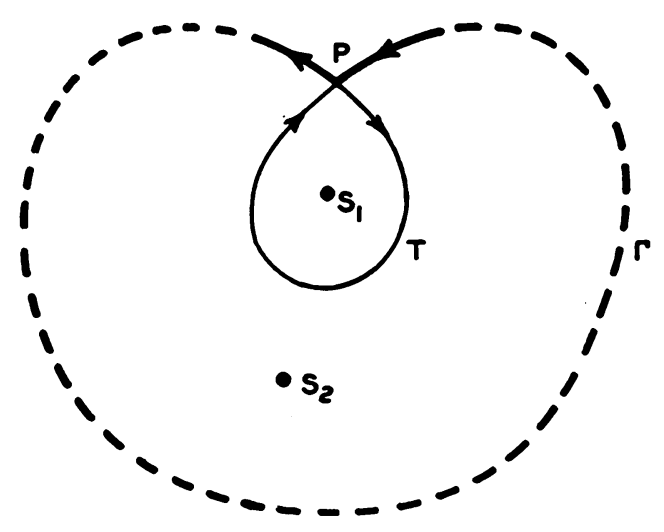

Fig. 3a.

region. Simple considerations, which we omit for the sake of brevity, show that the arc of trajectory cannot approach asymptotically to $\Gamma$. It follows, therefore, that the arc of trajectory must proceed to one of the points $S_{1}, S_{2}$, say the point $S_{1}$. Similar reasoning shows that the arc of trajectory which proceeds to $P$ must proceed from $S_{2}$.

We have the situation illustrated in Fig. $3 \mathrm{~b}$, which is drawn on the basis of the

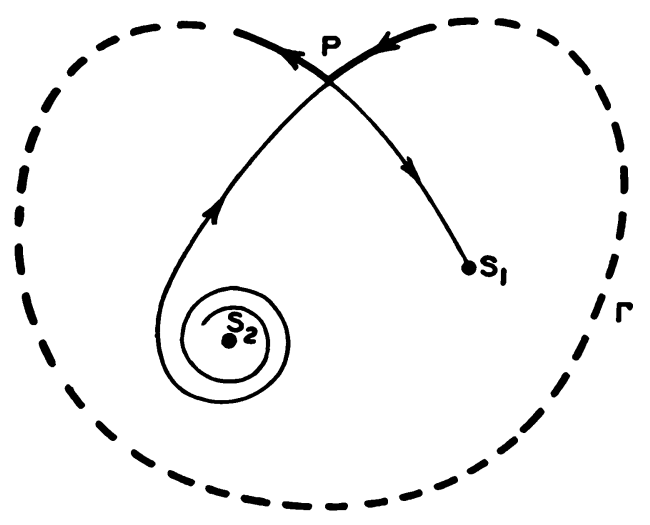

FIG. 3b.

assumption that $S_{1}$ is a node and $S_{2}$ a focus. The appropriate modifications of the figure for the cases in which $S_{1}$ and $S_{2}$ are of other types are obvious.

We note that in this second subcase $S_{1}$ and $S_{2}$ must be stable and unstable, respectively. 


\section{Case 2.}

Suppose that we have a p.c.t. $\Gamma$ with just two interior vertices, $P_{1}$ and $P_{2}$. Suppose also that the interior region determined by $\Gamma$, which is understood to belong to $R$, contains no centers, saddle points, or closed trajectories. Then the fundamental theorem implies that there are just three singular points, $S_{1}, S_{2}$, and $S_{3}$, in the interior region. Any one of these may be either a node or a focus. For the sake of simplicity we draw

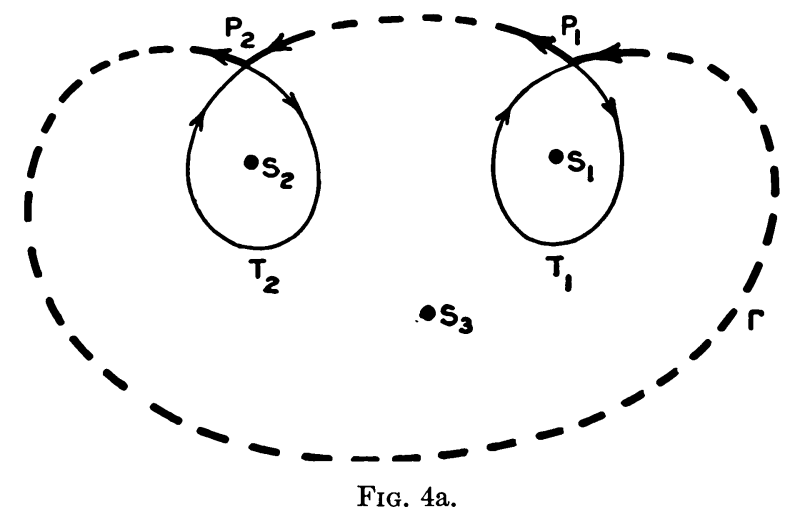

the figures for the case in which all three of these singular points are nodes. The modifications of the figures for the other cases are obvious. We shall see that when the types of $S_{1}, S_{2}, S_{3}$ are determined there remain six different possibilities as to the topological properties of the family of trajectories in the interior region.

1. Consider the two arcs of trajectories, in the interior region, which proceed from $P_{1}$ and $P_{2}$, respectively. It may be that the arc proceeding from $P_{1}\left(P_{2}\right)$ and the arc of trajectory proceeding to $P_{1}\left(P_{2}\right)$ belong to the same trajectory $T_{1}\left(T_{2}\right)$. This is the situation illustrated in Fig. 4a. Reasoning which is similar to that employed in the preceding

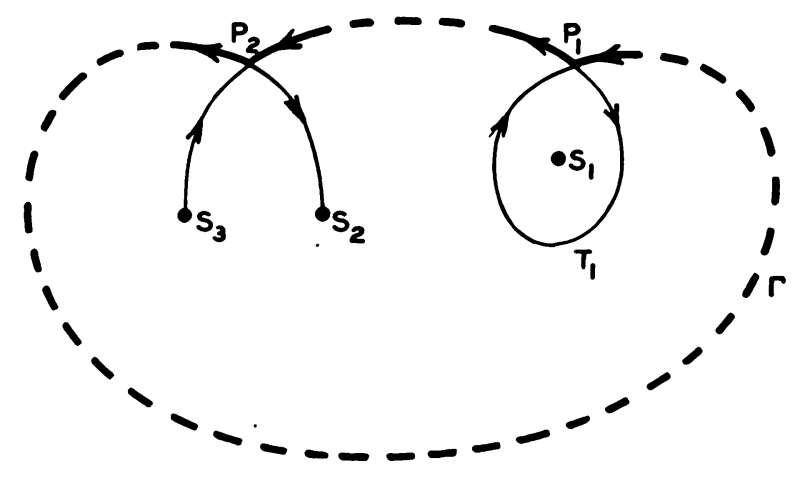

FIG. 4b.

case shows that if the subscripts are assigned suitably, the singular points $S_{1}, S_{2}, S_{3}$ must be located as shown in the figure.

2. It may be that the arc of trajectory proceeding from $P_{1}$ and the arc of trajectory proceeding to $P_{1}$ belong to the same trajectory $T_{1}$, but that the like is not true of the 
arcs of trajectories proceeding from and to $P_{2}$. Then one of the singular points $S_{i}$, say the point $S_{1}$, must be located in the interior region determined by the p.c.t. $\Gamma^{\prime}$ formed by $T_{1}$ and $P_{1}$. Also, we see that the arc of trajectory proceeding from $P_{2}$ must proceed to $S_{2}$ or $S_{3}$ say to $S_{2}$, and that the arc of trajectory proceeding to $P_{2}$ must proceed from $S_{3}$. This situation is illustrated in Fig. $4 \mathrm{~b}$.

3. It may be that the arc of trajectory which proceeds from $P_{1}$ proceeds to $P_{2}$, and that the arc of trajectory which proceeds from $P_{2}$ proceeds to $P_{1}$. This situation is illustrated in Fig. 4c. The fundamental theorem implies that the points $S_{1}, S_{2}, S_{2}$,

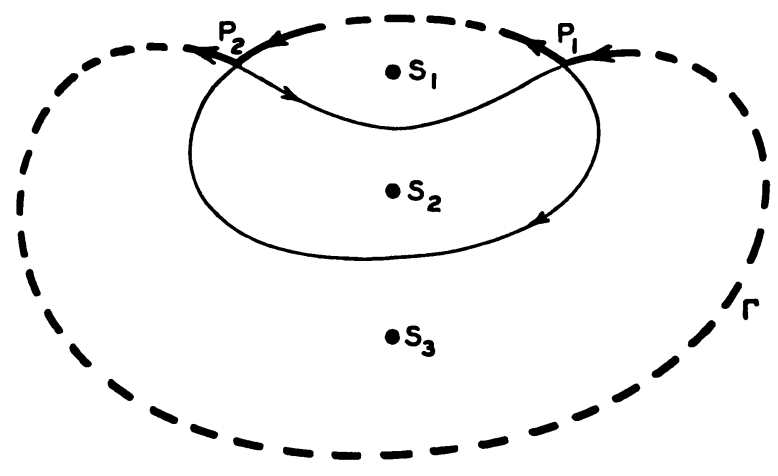

FIG. 4c.

with a suitable assignment of the subscripts, must be located with respect to the trajectories as shown.

4. It may be that the arc of trajectory which proceeds from $P_{1}$ proceeds to one of the singular points $S_{i}$, say the point $S_{1}$, and that the arc of trajectory which proceeds from $P_{2}$ proceeds to $P_{1}$. Then it is easily seen that the arc of trajectory which proceeds to $P_{2}$ must proceed from another singular point, say $S_{2}$. The third singular point must be located as shown in the illustrative Fig. $4 \mathrm{~d}$.

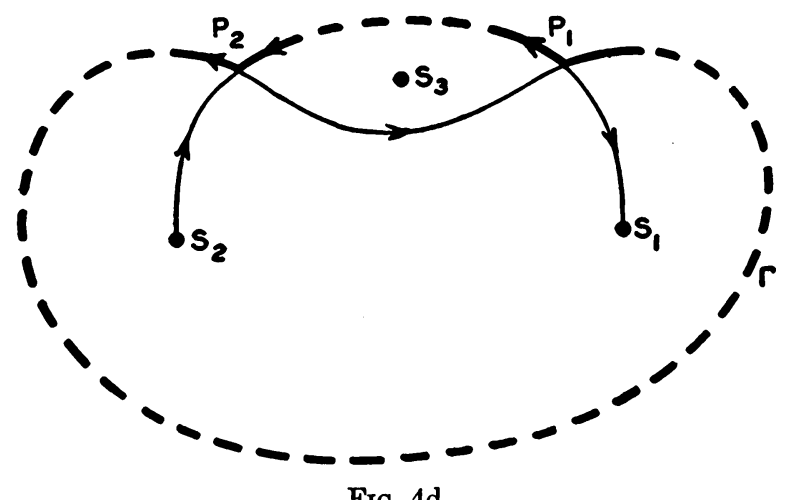

FIG. 4d.

5. In each of the four preceding subcases we have at least one arc of traectory which proceeds from one of the vertices $P_{1}, P_{2}$ to the same or the other vertex. Now suppose that this condition is not satisfied. Then the arcs of trajectories which proceed from $P_{1}$ and $P_{2}$ must proceed to the same or different ones of the points $S_{i}$. Suppose that the 
two arcs proceed to the same singular point $S_{3}$. It is clear that the arcs of trajectories proceeding to $P_{1}$ and $P_{2}$, respectively, must lie on opposite sides of the $\operatorname{arc} P_{1} S_{3} P_{2}$. Consequently, these arcs of trajectories must proceed from singular points $S_{1}$ and $S_{2}$, which are located as shown in the illustrative Fig. 4e.

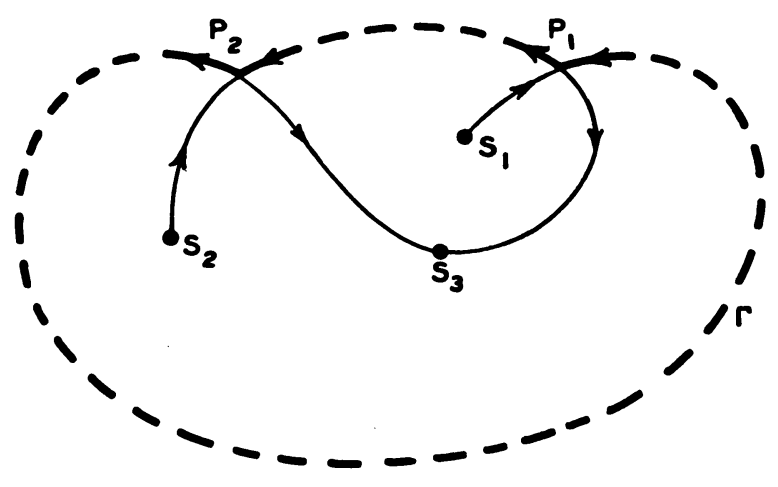

Fig. 4e.

6. Suppose, on the contrary, that the arcs of trajectories proceeding from $P_{1}$ and $P_{2}$ proceed to $S_{1}$ and $S_{2}$, respectively. Then it is easily seen that the arcs of trajectories proceeding to $P_{1}$ and $P_{2}$ must proceed from $S_{3}$. This subcase gives us a variant of Fig. $4 \mathrm{e}$ which we do not need to exhibit.

It is readily seen, in the light of the familiar Poincare theory of families of trajectories, that our discussion of the cases we have considered is essentially complete topologically. 